\title{
Folliculitis and scarring after eyebrow transplantation: an uncommon complication
}

\author{
Jocelyn Theresa P. Navalta, MD, Damkerng Pathomvanich, MD, FACS Bangkok, Thailand jocelynnavalta@ymail.com
}

We report a case of recalcitrant folliculitis resulting in atrophic scarring after eyebrow transplantation. Folliculitis, a common complication in hair transplant surgery, is an uncommon occurrence in eyebrow transplantation for unknown reasons. The various factors possibly affecting the eruption of folliculitis in this case are: 1) dense packing, 2) skin type and pre-existing lesions, 3) hypersecretion of sebaceous glands, and 4) postoperative care. Atrophic scarring is a consequence that may still be managed with various dermatologic treatments such as chemical peels and other skin resurfacing procedures.

\section{Introduction}

Folliculitis is a common complication from hair transplant surgery, second only to widened donor scars, ${ }^{1}$ that typically occurs 1 month after surgery and may last up to 6 months. ${ }^{2}$ These inflammatory, often pruritic papules and pustules are caused by bacteria (Staphylococcus aureus) and are thus treated with topical (Mupirocin) and/or antibiotics (Tetracyclines), warm compresses, and antiseptics such as chlorhexidinegluconate (hibiclens), hexachlorophene (phisohex), and povidone-iodine (betadine) shampoo. We rarely perform cultures since folliculitis is usually superficial and mild, and resolves spontaneously with or without medical management. With deep recurrent or more resistant cases, however, other etiologies should be considered such as gram-negative bacteria (Pseudomonas, Enterobacter, Proteus, and Klebsiella), fungal infection (Malassezia), or viral infection (Herpes simplex), and in severe cases MRSA. ${ }^{2,3}$

Folliculitis has been attributed not only to microbial etiologies but also to the inappropriate depth of insertion causing burial of grafts, ${ }^{4,5}$ to the "piggy-backing" of grafts, ${ }^{2,5}$ and to a high density of the transplanted grafts (more than $30-35 \mathrm{FUs} / \mathrm{cm}^{2}$ ). It has been proposed that extreme densities cause an increase in number of sebaceous glands transplanted along with the grafts, which are unregulated and over produce sebum. ${ }^{6}$ Other factors noted to increase the occurrence of folliculitis include scalp oiliness, preexisting inflammatory scalp lesions, dusty work environments, infrequent scalp washing, or immunosuppressive states such as diabetes mellitus. ${ }^{7,8}$

Eyebrow transplantation can have folliculitis as a complication for similar reasons as in scalp transplantation, although occurrences seem to be rarer. We present a case of folliculitis after eyebrow transplantation resistant to usual antibiotic management that eventually led to atrophic scarring.

\section{Case Study}

A 35 year-old Asian male consulted for hair transplantation due to mild frontotemporal recession with androgenetic alopecia Norwood II classification and diffusely sparse eyebrows (Figure 1). Prior to surgery, he had minimal erythematous patches over both medial eyebrows and few closed comedones on the forehead diagnosed as mild seborrhea and mild acne, respectively. The patient had been using various topical medications to manage his oily skin and acne eruptions.

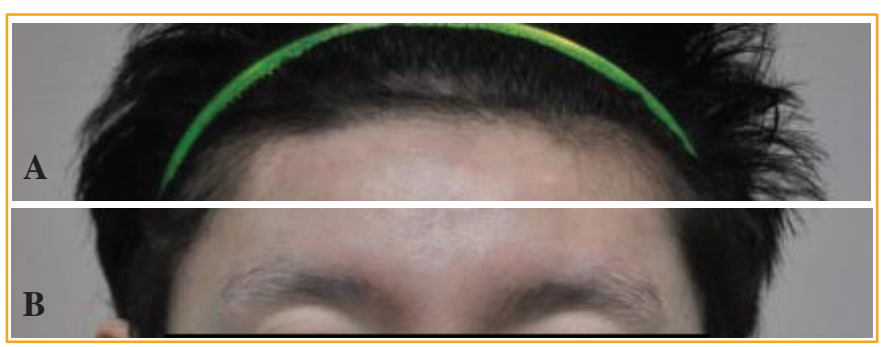

Figure 1. A: Pre-op: Mild frontotemporal recession. B: Pre-op: Bilateral sparse eyebrows.

The patient underwent hairline and eyebrow transplantation and had a total of 2,007 grafts and 3,782 hairs (1-hair grafts $=559$, 2-hair $=1,167$, 3-hair $=235$, 4-hair $=46$ ) for hair transplantation and a total of 710 grafts for eyebrow transplantation (right eyebrow: 1-hair $=330,2$-hair $=25$; left eyebrow: 1 -hair $=330,2$-hair $=25$ ) . For his hairline, grafts were inserted into pre-made incisions using 18-21 gauge needles. For his eyebrows, stick-and-place technique with depth control was performed using 21 and 23 gauge bent needles. The patient was prescribed Doxycycline 100mg BID for 5 days and mild shampoo daily.

Two months after surgery, the patient reported (over the phone) to have eruption of multiple erythematous papules and pustules diffusely spread over the frontal hairline and eyebrows, for which topical antibiotic ointment (Mupirocin) was advised twice a day with report of some improvement. Upon follow-up at the clinic, he was found to have several erythematous papules on the frontal hairline, enlarged pores over inflamed skin, and comedones on both medial eyebrows (Figure 2). He was advised to use the following medications: Fusidic acid with Hydrocortisone cream BID, Ciprofloxacin 500mg BID for a week, and a mild shampoo for daily cleansing.

Three months after surgery, there was a decrease

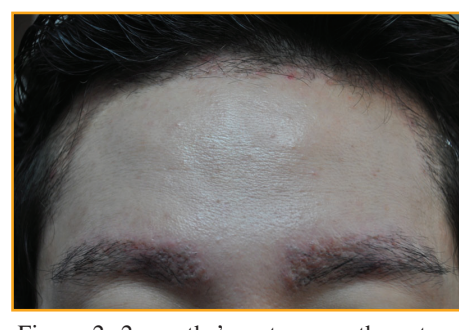

Figure 2. 2 months' post-op: erythematous papules (hairline and eyebrows), comedones and enlarged pores (eyebrows). in erythema and papules at the hairline and atrophic scar formation at the medial eyebrow (Figure 3). He was advised to take Doxycycline 100mg BID for 1 month, Metronidazole gel BID, Tretinoin $0.5 \%$ gel OD, Betamethasone cream BID, and Nizoral shampoo daily (left on for 5 minutes before rinsing).

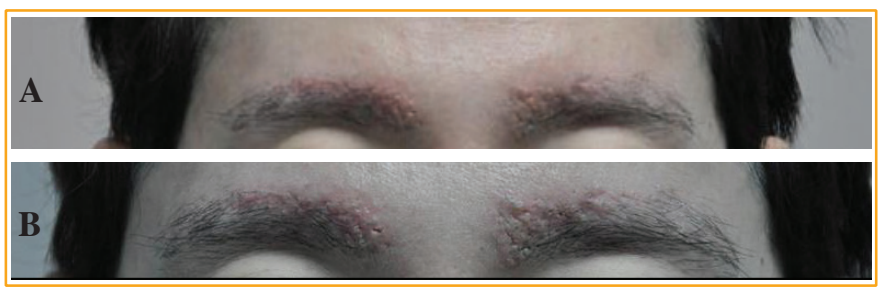

Figure 3. A: 3 months' post-op: decrease in erythema and number of papules. $B: 3$ months' post-op: atrophic scarring.

At 4-month follow-up post-transplantation, there was resolution of papules and pustules over the hairline and some improvement over the eyebrows (Figure 4). Minimal erythema 
(attributed to topical retinoids) and atrophic ice-pick shaped scars remained over the medial eyebrows (Figure 4C). Open comedones were extracted and the patient was recommended topical Benzoyl peroxide in combination with Adapalene and to undergo skin-resurfacing treatment with a

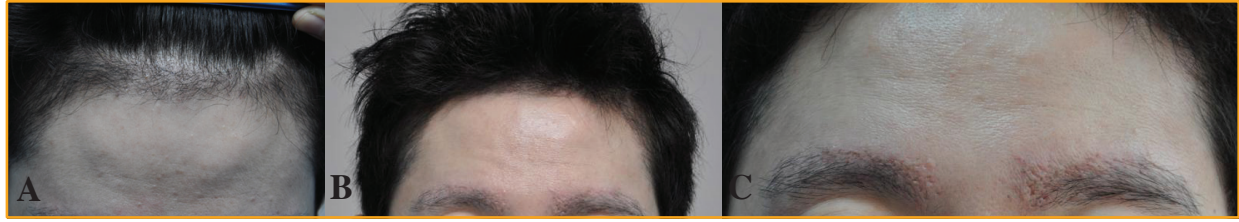
Figure 4. A: 4 months' post-op: resolution of lesions (hairline). B: 4 months' post-op: improvement of lesions (eyebrows). $C: 4$ months' post-op: increase in "ice-pick" (atrophic) scars.

Dermatologist. The patient underwent several weekly TCA chemical peel treatments with gradual improvement of his scars (Figure 5). Hair growth at the medial aspect of both eyebrows had been minimal but signs of regrowth, though gradual, are promising.

\section{Discussion}

\section{Dense Packing and Overcrowding}

Reconstruction of eyebrows using micrografts usually produces good results. We use single-hair transplantation technique with meticulous dissection of grafts into ultraskinny single hairs with the aid of stereomicroscopes with $10 x$ magnification. Stick-and-place insertion using 23-25 gauge needles has been used in the past to provide dense packing with a survival rate of more than $80 \% .^{9}$ Stick-and-place technique using 21-23 gauge needles is now our preferred method for eyebrow transplantation, which allows for less dense packing and less need for ultra-skinny grafts without compromising cosmetic outcome.

The number of grafts per eyebrow ranged from 116-252 grafts for males and 130-215 grafts for females in the five eyebrow transplant cases among Asians we transplanted. ${ }^{9}$ The use of approximately 600 single-hair grafts in total for eyebrow reconstruction has also been reported..$^{10}$ Previously, we have utilized an average of 100-200 single-hair grafts on each eyebrow without complications. ${ }^{11}$ Most hair surgeons who do eyebrow transplantation usually insert between 200-300 single-hair grafts per eyebrow. In our case, a total of 710 grafts with approximately 350 grafts per eyebrow have been transplanted, including 252 hair grafts per side. The use of 2-hair grafts to add more density to the eyebrows may have promoted overcrowding.

It has also been suggested that transection of sebaceous glands during incisions in the recipient site to create slits, which releases sebum into the surrounding tissue, has caused cyst formation. ${ }^{11}$ This can occur more frequently during dense packing of grafts.

\section{Skin Type and Preexisting Lesions}

Skin type, such as oily or dry skin and whether preexisting sources of infection are present, should be included in the physical examination of patients. These factors may increase the risk of having a more severe eruption of folliculitis. Our patient had oily skin, mild erythema over both medial eyebrows (seborrhea), as well as small closed comedones on the forehead (acne) - factors that may have influenced the severity of the eruption of folliculitis.

\section{Hypersecretion of Sebaceous Glands}

We could not explain the occurrence of folliculitis using skinny grafts as they are almost devoid of surrounding tissue, including sebaceous glands. Possibly there is regeneration of these sebaceous glands, unregulated secretion of sebum, and an increase in the total number of sebaceous glands in the area.

\section{Post-Operative}

\section{Management and Care}

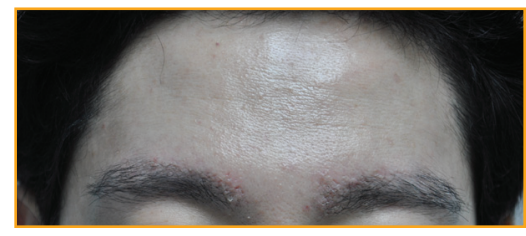

Figure 5. 5 months' post-op: improved scars with TCA peel.

Post-operative wound care reports have compared the use of dry and moist dressings with results showing no difference in growth and no reports of folliculitis as a complication. ${ }^{12}$ No occlusive dressing was applied in our case. Post-operative care also included preventive oral antibiotics and adequate daily cleansing. Subsequent folliculitis after failure to respond to empiric therapy in our case was treated with a combination of topical and oral antibiotics, topical anti-inflammatory steroids, and daily cleansing, though response to these treatments was slower than expected. A superimposed fungal infection from seborrhea or presence of gram-negative bacterial infection could have occurred, hence the possible resistance to usual grampositive oral and topical antibiotics. The addition of appropriate management was then included for complete coverage.

\section{Atrophic Scarring: Sequelae from Deep Infection}

Scarring from folliculitis occurs when the extent of the inflammation and infection is deep (dermal). Most deep folliculitis requires incision and drainage and a more broad-spectrum antimicrobial therapy. Eventual treatment of the atrophic scars is needed and may prove difficult since they are located in a hair-bearing region. Topical keratolytics (retinoids, chemical peels) and microneedling are several treatment options. Other modalities, such as microdermabrasion, and skin resurfacing lasers, such as ablative and non-ablative fractional lasers, ${ }^{13,14}$ also may be utilized. Weekly TCA chemical peels for our patient have been beneficial and have shown satisfactory results.

\section{Conclusion}

Folliculitis from eyebrow transplantation may be a rare complication, but it is important to anticipate its occurrence and be able to manage it appropriately to prevent eventual permanent scarring. The reason for its occurrence can be multifactorial. Factors to consider are dense packing, skin type, and presence of preexisting lesions, sebaceous gland hypersecretion, and the need for individualized appropriate post-operative management and care. Other considerations are the depth of graft insertion, the use of ultra-skinny grafts, and the "piggy-backing" of grafts (not considered in our case). Eventual skin resurfacing treatments should be offered if scarring occurs.

\section{References}

1. Salanitri, S., et al. Surgical complications in hair transplantation: a series of 533 procedures. Aesthet Sugr J. 2009(JanFeb); 29(1):72-76. 
Folliculitis

from page 55

2. Mejia, R. Folliculitis. Hair Transplant Forum Int'l. 2008; 18(5):183-184.

3. Perez-Meza, D. Day-by-day review of the 2011 ISHRS 19th Annual Scientific Meeting. Hair Transplant Forum Int'l. 2011; 21(6):196.

4. Breeling, J.L, J.C. Kim. Improving survival of follicular unit grafts. Hair Transplant Forum Int'l. 2004; 14(4).

5. Marzola, M., and J.E. Vogel. (2006). Complications. In: R.S. Haber and D.B. Stough, eds. Hair Transplantation (173-185). China: Elsevier Saunders.

6. Tykocinski, A. A super-megassession of 2,800 to 4,000 follicular units, packed to $40.5 \mathrm{FUs} / \mathrm{cm}^{2}$ : Are you prepared? Hair Transplant Forum Int'l. 2006; 16(5):153.

7. Bunagan, M.J., D. Pathomvanich, and K. Laorwong. Recipient area folliculitis after follicular-unit transplantation: characterization of clinical features and analysis of associated factors. Dermatol Surg. 2010; 36(7):1161-1165. Epub 2010 Jun 1.
8. Nusbaum, B.P., and A.G. Nusbaum. (2011). Recipient area complications. In: W. Unger and R. Shapiro, eds. Hair Transplantation, 5th edition (422-424). United Kingdom: Informa Healthcare.

9. Laorwong, K., M.J. Bunagan, and D. Pathomvanich. Eyebrow transplantation in Asians. Dermatol Surg. 2009; 35:496-504.

10. Kim, J.C., and Y.C. Choi. Hair Replacement: Surgical and Medical. St. Louis Missouri: Mosby.1996; 216-218.

11. Marzola, M. Cyberspace Chat: Cysts in the recipient site. Hair Transplant Forum Int'l. 2001; 11(5):146.

12. Pathomvanich, D. Anatomical Restoration Surgery of the Eyebrow in Asian. The Thai J Surg. 2004; 25:47-52.

13. Alajian, A.M., and S.N. Alsuwaidan. Acne scars in ethnic skin treated with both non-ablative fractional $1,550 \mathrm{~nm}$ and ablative fractional $\mathrm{CO} 2$ lasers: comparative retrospective analysis with recommended guidelines. Lasers Surg Med. 2011(Sep); 43(8):787-791.

14. Qian, H., et al. Treatment of acne scarring with fractional CO2 laser. J Cosmet Laser Ther. 2012(Jun 6). [Epub ahead of print].

\section{The New Face of ISHRS.org}

We are very excited to announce the rollout of our new ISHRS.org website! There have been several enhancements to the new site including:

- Featured Videos: Over the next several months, we will be rolling out over 30 new consumer-focused videos talking about a number of hair loss and restoration subjects.

- New, Fresh Content: The ISHRS.org homepage now features brief, ongoing articles pertaining to hair loss treatment and restoration that will be updated on a weekly basis.

- Discussion Forum: We have created a place for patients, prospective patients, and each of you to collaborate-posting comments about the topics and issues that impact anyone experiencing hair loss.

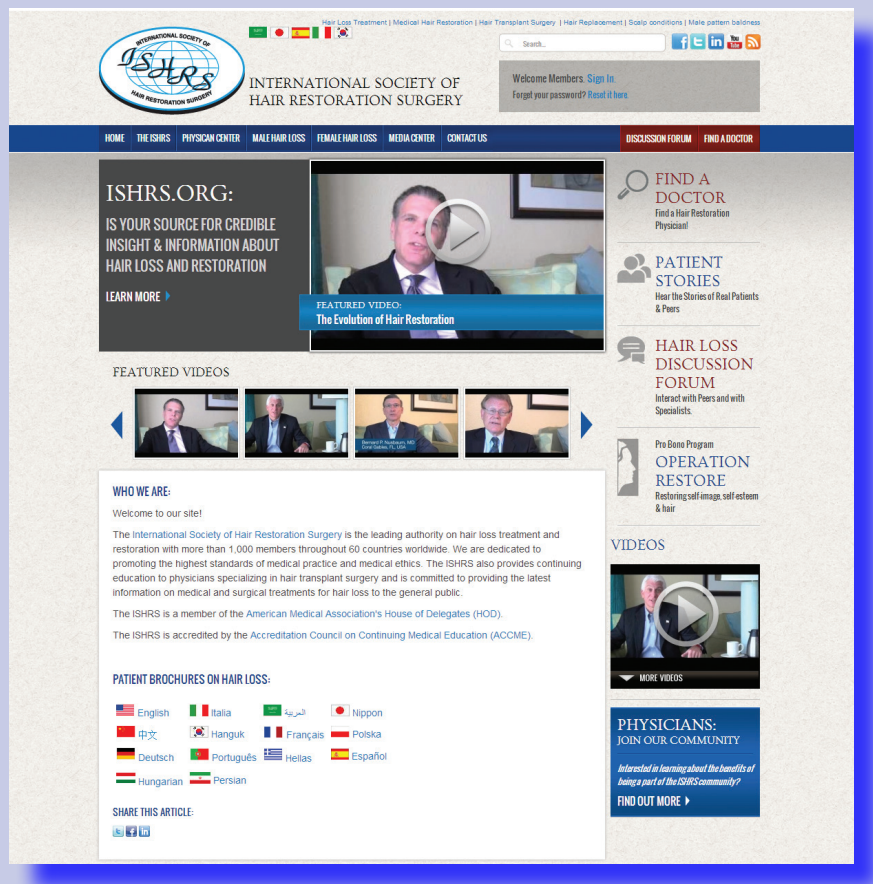

- New \& Improved "Find A Doctor" Section: We have improved the search functionality and features of our "Find A Doctor" section allowing consumers to more quickly access our physician directory and search by keyword (e.g., address, last name, practice areas, etc.).

Each Physician Member has received a welcome email introducing the new site and providing personal, new login information for access to the "Member's Only" section. Instructions are included and members are urged to verify personal information displayed in the "Find A Doctor."

We look forward to your feedback about the new site. Please don't hesitate to contact Matt Batt, ISHRS Integrated Communications Manager (mbatt@ishrs.org) with any questions or feedback. 